\title{
The effect of the relevancy of an observation stimulus on second learning in concept identification
}

STANFORD H. SIMON AND BASIL JACKSON

V. A. CENTER, WOOD, WISCONSIN AND MARQUETTE UNIVERSITY SCHOOL OF MEDICINE

Three groups of Ss, 12 each, learned two concept identification (CI) tasks. In the first task there was no observation stimulus $\left(S^{0}\right)$. In the second task, one group had a relevant $S^{\circ}$, a second group had an irrelevant $S^{\circ}$, and a third group had no $S^{0}$. The so significantly affected CI learning as a direct function of the relevancy of the $S^{o}$, even though $S s$ had become familiar with the task involved.

In an earlier study (Simon \& Jackson, 1967) the authors found that a relevant observation stimulus (RSO) significantly facilitated learning and that an irrelevant observation stimulus $\left(15^{\circ}\right)$ significantly retarded learning, when compared to a no-observation stimulus (NS) condition. The learning involved an initial concept identification task. The present study seeks to answer the following questions. After $\mathrm{S}$ has "learned how to learn" with an initial task, would the introduction of an $\mathrm{RS}^{\circ}$ tend to distract from learning rather than to facilitate it? Will knowing how to solve the concept identification task without the aid of an $S^{\circ}$ tend to negate the effect of an ISO? It is the purpose of this investigation to study the effect of the relevancy of an $\mathrm{S}^{\circ}$ on second learning in concept identification.

Method

All Ss learned two concept identification tasks presented on two decks of cards. The procedures and the first task were identical to those used in the previous study (Simon \& Jackson, 1967). Briefly, it consisted of two dimensions defined by the letters " $d$ "' and " $h$, " with two levels to each dimension, defined as the upper and lower case of the letters. One level of each dimension was presented on each card; the positions of the letters relative to each other were systematically varied. This provided eight combinations, presented in random order in each of four replications, for a total of 32 cards.

The second task was similar to the first, except that it involved three dimensions defined by the letters " $q$, " " $r$," and " $t$ " with the same two levels to each dimension. There were 48 cards presented in random order.

For all Ss the relevant dimension in the first task was "'d"; in the second task it was "t." In both tasks, $S$ had to identlfy the cards as belonging to "Group $1 "$ or "Group 2." Cards with the upper case of the relevant dimension belonged in Group 1, those with the lower case belonged in Group 2. The correct answer was written on the back of each card; $S$ recorded his guess, turned the card, and recorded the correct answer. As in previous studies (e.g., Simon, 1962), a dot over a stimulus dimension served as an $\mathrm{S}^{\circ}$.

Three groups of $12 \mathrm{Ss}$ each were used. The NS group had no $\mathbf{S}^{\circ}$, the $\mathrm{RS}^{\circ}$ group had a dot over the "t" dimension, the ISo group had a dot over the " $q$ " dimension. All Ss had no so during the initial task.

The Ss were introductory psychology students. They were tested in groups of up to nine, with each condition represented equally. The Ss were randomly assigned to the groups.

\section{Results and Discussion}

The first question raised is whether Ss "learned to learn" on Task 1 . Since all Ss had the NSO condition during Task 1 , independent estimates of the number of errors in Task 1 and Task 2 could be obtained. This was done by combining the error scores on Task 1 for the $I S^{\circ}$ and $R S^{\circ}$ groups $(N=24)$ and comparing them with the first 32 trials of Task 2 for the NSo group $(\mathrm{N}=12)$. Both comparisons, therefore, are under the $\mathrm{NS}^{\circ}$ conditions and each have 32 trials. In this comparison the Task 1 group had $\mathrm{m}=9.63, \mathrm{SD}=6.36$; the Task 2 group had $m=4.92, S D=4.87$. Task 2 produced significantly fewer errors $(t=2.188$, df $=34, p$ $<.05)$, in spite of the fact that Task 2 had one irrelevant dimension more than was present in Task 1.

In Task 2, using all 48 trials, the means (and SDs) of the three groups were: $\mathrm{RS}^{\circ}, 3.08$ (3.97); NSO 6.75 (8.21); IS $^{\circ} 10.83(9.00)$. The means in the present study have the same relationship as in the previous study. Using a pooled MS error score (repeated measures, six blocks of eight trials each) of 9.96, we find that the RSO significantly facilitates learning (RSO vs $\mathrm{NS}^{\circ}, \mathrm{t}=2.844$, df $=33, \mathrm{p}<.01$ ), the ISo significantly retards learning (ISO vs $\mathrm{NS}^{\circ}, t=3.162$, of $=33, p<.01$ ), and the two conditions, $\mathbf{R S}^{\circ}$ and $I S^{\circ}$, differ significantly $(t=6.023, d f=33, p<.001)$.

While the number of errors is significantly affected by the $\mathrm{RS}^{\circ}$ and IS , all three groups on Task $2 \mathrm{had}$ more Ss master the task than those in the previous study exposed only to Task 1 , again reflecting the learning efficiency gained by having an initial task. Considering only the first 32 trials, and using as a criterion of learning one complete block of eight trials without error, the present study (and previous study) had the following percentages of $\mathrm{S}$ reach criterion: $\mathrm{RS}^{\circ}, 1.00$ (.66); $\mathrm{NS}^{\circ}, .83$ (.37); IS ${ }^{\circ}, .67$ (.34). 
In every instance, the Task 2 group had more Ss reach criterion.

With the finding that an so continues significantly to affect concept identification learning as a direct function of the relevance of the $S^{\circ}$, even after $S s$ have become familiar with the tasks involved, this study replicates and extends the findings of the previous study.

\section{References}

Simon, S. H. Effect of a relevant observation stimulus on human discrimination learning as a function of the position of the relevant stimulus. Percept. mot. Skills, 1962, 15, 359-365.

Simon, S. H., \& Jackson, B. The effect of a relevant vs. irrelevant observation stimulus on concept identification learning. $J$. exp. Psychol., 1967, in press. 